\title{
Development of an Hexapod BioMicroRobot with Nafion-Pt IPMC Microlegs
}

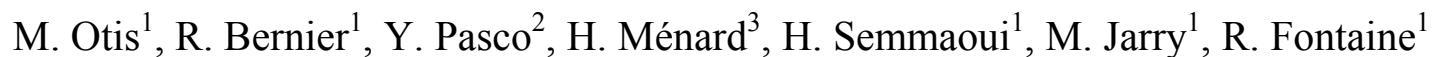 \\ ${ }^{1}$ Department of Electrical Engineering and Computer Engineering, University of Sherbrooke, Québec, Canada \\ ${ }^{2}$ Department of Mechanical Engineering, University of Sherbrooke, Québec, Canada \\ ${ }^{3}$ Department of Chemical, University of Sherbrooke, Québec, Canada
}

\begin{abstract}
This paper presents an artificial locomotion servo-system for an insect like hexapod BioMicroRobot (BMR). This servo-system, programmed with VHDL code, will act as a driver in a RISC architecture microcontroller to reproduce insect tripod walking. An overview of the robot control system, in accordance with the insect displacement principle, is demonstrated with timing parameters. A control algorithm of the six legs driving the robot in any direction versus Pulse Width Modulation (PWM) is reviewed. BMR microlegs are built with cylindrical Nafion-Pt Ionomeric Polymer-Metal Composite (IPMC) that have 2.5 degrees of freedom. Specific fabrication process for one leg is exposed. Dynamic behavior and microleg characteristics have been measured in deionized water using a laser vibrometer. BMR current consumption is an important parameter evaluated for each leg. Hardware test bench to acquired measurement is presented. The purpose of this design is to control a BMR for biomedical goals like implantation in human body. Experimental results on the proposed legs are conclusive for this type of bioinspired BMR.
\end{abstract}

Keywords-IPMC, locomotion, Nafion, biomicrorobot.

\section{INTRODUCTION}

Several designers and researchers in micro and nano robotic hope their robots will perform biomedical applications. Currently, these robots are at the early stage of development and the objectives to reach are spread out over several years [1]. Many microactuators are in development for insect-like microrobot legs such as bimorph cantilever structures acting as thermal actuator coupled or not with Photonic Band Gap (PBG) materials, piezoelectric, electrostatic, magnetostrictive, shape memory alloy (SMA) and ElectroActive Polymer (EAP) like ionic polymer to name a few [2].

The BioMicroRobot (BMR) actuator consist in a cylindrical Nafion-Pt Ionomeric Polymer-Metal Composite (IPMC). IPMC has three components: an Ion-Exchange Membrane (IEM) like perfluorosulfonic acid polymer, some cations and at least two electrodes. This EAP actuator has many advantages over all others: has quick response to low voltage, bends more than $100 \mathrm{~K}$ times, generates distributive deformation, has a small weight, can be downsized easily and can be used in water [3]. It seems to be safe in human body [4] while no water electrolysis is produced. It can be driven at low voltage; less than 2 Volts but step transient response shows high instantaneous current consumption with Nafion-Pt and Lithium counter-ion. (around $\mathrm{mA}$ ). Water electrolysis and current consumption are two major IPMC disadvantages for microrobot applications.
IEM consist of a cross-linked ionomer matrix with a relatively uniform distribution of ion-active sites (cluster network) throughout the structure. Effects of different types of cations, electrode-membrane interfacial area, and surface electrode resistance on performance are well known [5], [6] and [7]. IPMC works on two principles. First, the electroosmosis in the ionomer moves the cation and water to the cathode side by the electrostatic force when an electric field is applied. The osmotic pressure inside the polymer creates bending moment that produces a corresponding displacement [8]. The second principle is pumping effect of cations. It would be the dominant contribution in the case of hydrophobic cations [9].

\section{Methodology}

In previous study, we have used tubular Perma Pure Inc. TT-030 Nafion ${ }^{\circledR}$. However, actuator movement is too small for this application due to its shape. Actually, the use of Nafion ${ }^{\circledR}$ N-117 (DuPont) fiber is better for displacement rate and current consumption. The size of this new microactuator is about $200 \mu \mathrm{m} \times 200 \mu \mathrm{m}$. Its length $\mathrm{L}$ is about $3000 \mu \mathrm{m}$ for the actuator characterization (Fig. 2). Expected final microlegs length on BMR body is about 1500 $\mu \mathrm{m}$.

\section{A. IPMC Fabrication}

This method can be done with any ionomer membrane. Ion Exchange Membrane (IEM) is a hydrophilic ionic polymer and swells up about $10 \%$ in water at room temperature. The membrane N-117 is cutted in fibers of 187 $\mu \mathrm{m}$. Fibers are attached to a rotating device and are roughened at about 150 RPM using smooth sandpaper 1000 . The ionomer is then cleaned in an ultrasonic water bath and boiled in $\mathrm{HCl}$ and water about 30 minutes each. The ionomer is chemically plated with platinum [10]. Ionexchange (absorption) and reduction process are repeated up to two times to make thick porous dendrilic platinum layers. $\mathrm{Li}+$ cations are used on all the IPMC to get fastest responses. The electrodes on the tube and fiber are cut in four sections using a laser process (Fig. 6a).

\section{B. Microleg experimental measurement}

In order to obtain the dynamic behavior of a microlegs under electrical solicitation, a scanning laser vibrometer is used to measure the free end speed of displacement. 


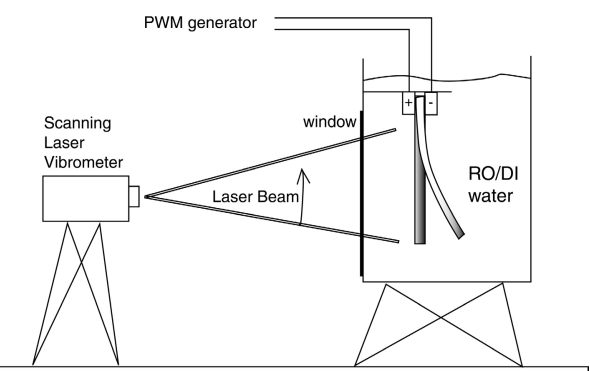

Fig. 1. Experimental setup for speed measurement of the microleg.

The beam formed by the actuator is clamped at one end and is acting freely in deionized water. The laser beam goes through an optical window and reaches the microleg perpendicular to its neutral plane (Fig. 1). Refraction of light is leaved out in consideration of relative measurements.

The applied voltage is a periodic Pulse Width Modulation (PWM) signal programmed in an arbitrary waveform generator HP33120A. The waveform consists of a 1024 points signal, where half points form a positive PWM and the others a negative one. Instantaneous speed was measured at the tip of the actuator and stored for all duty cycles used in the PWM signals of the waveform. The waveform has a $3 \mathrm{~V}$ peak to peak and is applied at 10 and 20 $\mathrm{Hz}$. Those measurements were done far away from the first resonance of the mechanical system: a modal analysis of the microleg using a random applied voltage and scanning capability of the vibrometer reveals a first natural frequency of $180 \mathrm{~Hz}$. This frequency takes into account coupling between the fluid and the structure.

\section{Microlegs mathematical modelisation}

Behavioral simulation is useful to predict microlegs movement. Simulation coupled with scanning laser vibrometer measurements orient the design of a bioinspired control algorithm. Theoretical equations in cylindrical coordinate must be defined to predict the microlegs movement. Two conditions have been assumed to obtain theoretical equations on 2D bending characteristic. The actuator bends in a certain curvature (a circular arc model) and the length of neutral plane is fixed. 2D equations in universal rectangular coordinates for an active catheter are presented in [11]. Fig. 2 shows the bending principle. The actuator of length $\mathrm{L}$ bends at angle $\alpha_{\mathrm{i}}$ around a circle of center $\mathrm{O}$. When the IPMC bends, the tangent circle of rayon $r_{i}$ decreases with time. The bending angle $\alpha_{i}$ and displacement $s_{i}$ depend on the voltage $V_{i}$ applied to the electrode. Tip position $\left(\mathrm{x}_{\mathrm{i}}, \mathrm{y}_{\mathrm{i}}\right)$ can be calculated with (1) if $\mathrm{z}$ $=0$. For this application, equations are showed in cylindrical coordinates $\left(\rho_{\mathrm{i}}, \theta_{\mathrm{i}}, \mathrm{Z}_{\mathrm{i}}\right)$.

$$
\begin{gathered}
x_{i}=r_{i} \sin \left(\alpha_{i}\right) \\
y_{i}=\operatorname{sqrt}\left(r_{i}^{2}\left(1-\cos \left(\alpha_{i}\right)\right)^{2}-z_{i}^{2}\right) \\
\rho_{i}=2 r_{i} \sin \left(\alpha_{i} / 2\right) \\
\theta_{i}=\alpha_{i} / 2=L /\left(2 r_{i}\right)
\end{gathered}
$$

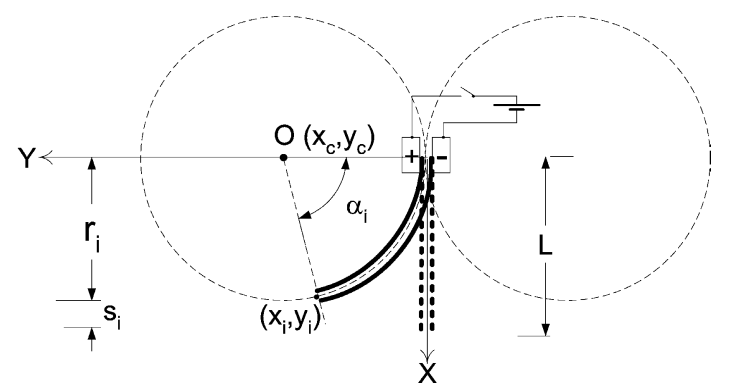

Fig. 2. Bending principle of IPMC.

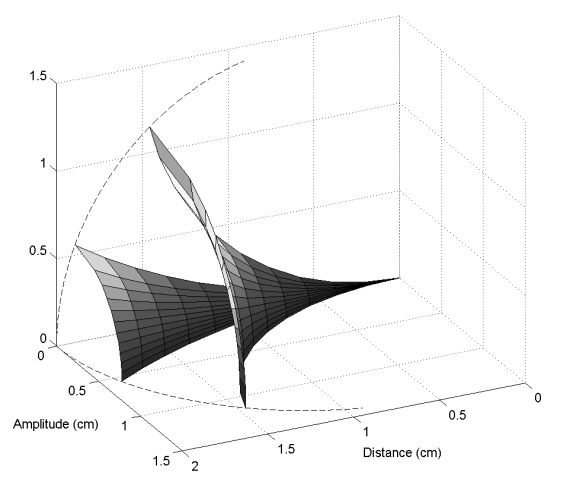

Fig. 3. 3D leg movement simulation with two different voltages.

Fiber actuators have four identical electrodes as depicted in Fig. 6a so they can bend in 2,5 DOF. The control algorithm will generate eight states $S_{i}$ to move the actuator in any direction. Based on the theoretical equations developed previously, Fig. 3 shows simulation of two steps length versus the applied voltage ( 1 and 3 Volts) and the eight states. IPMC is held at coordinate $(0,0,0)$. Next section demonstrates that simulation to the insect behavior allows the robot to turn in any direction when the applied voltage of one BMR side changes.

\section{BIOINSPIRED CONTROL ALGORITHM}

IPMC mechanical properties can be used to drive BMR like an insect. Hexapod insect walking shows who the control algorithm must be designed to drive each microlegs with great stability.

\section{A. Hexapod Insect Walking}

The cyclic movement of a walking leg consists of two parts (Fig. 4): the power stroke (PS, also stance or support phase that propels the body) and the return stroke (RS, also swing or recovery phase). The anterior transition point (transition from swing to stance) is called the anterior extreme position (AEP) and the posterior transition point is called the posterior extreme position (PEP). The most critical point to maintain robot's stability is the transition from PS to RS [12]. 


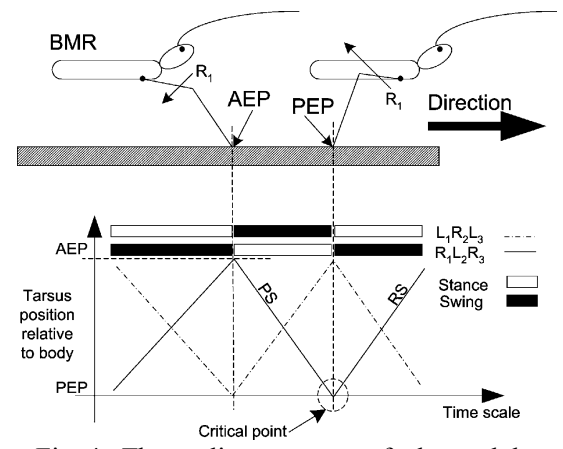

Fig. 4. The cyclic movement of a leg and the tripod (fastest gait) walking stepping pattern.

Two conditions must be reached to keep robot's stability at all locomotion phases. The center of gravity of the body must lie in the triangle of support. Also, three feet must always be on the ground simultaneously. The body is supported alternatively, for equal period of time, by one of the two leg groups (tripod gait): $A\left(\mathrm{~L}_{1} \mathrm{R}_{2} \mathrm{~L}_{3}\right)$ or $\mathrm{B}\left(\mathrm{R}_{1} \mathrm{~L}_{2} \mathrm{R}_{3}\right)$ (Fig. 5). The stability margin is defined as the shortest distance $\left(d_{1}, d_{2}, d_{3}\right)$ from the center of mass to the edge of the tripod of support. Many insects closely conform to this pattern when moving quite rapidly [13], [14]. Two main strategies are studied to allow the insect to turn: increasing step frequency or step length of legs on one side of the body versus the other [15].

\section{B. Microlegs configuration}

BMR body is the support for the actual microlegs. Leg configuration on body is an important consideration to drive the robot in any direction (Fig. 6b). Experimental results show that half period of stance doesn't need any power consumption while the other legs are in swing period (maximal current consumption). IEM elasticity propels the BMR body. Bending angle decrease or increase the stance and the swing time that produce BMR turn.

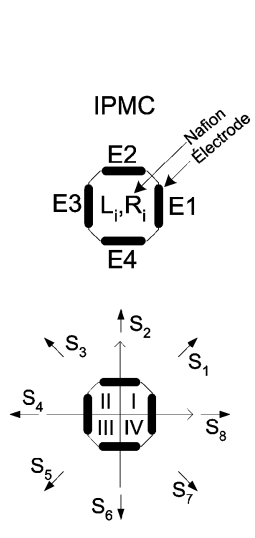

(a)

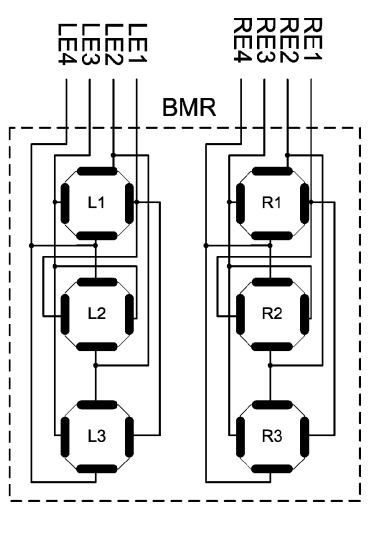

(b)
Fig. 6. (a) Leg cross-section with four electrodes in Cartesian coordinate. The states $\mathrm{S}_{\mathrm{i}}$ generate bending moments and the associated direction movement. (b) BMR body hardware.

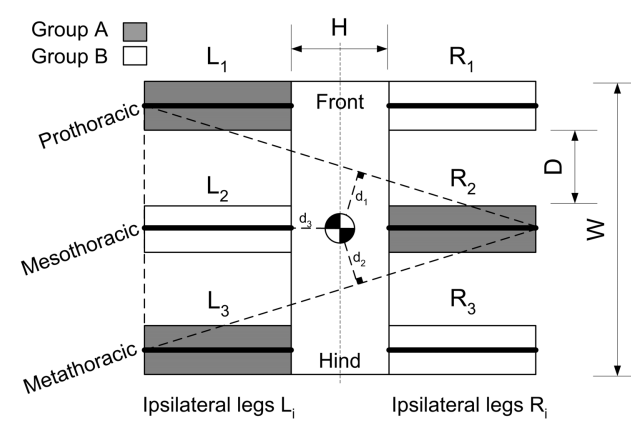

Fig. 5. Upper view of microrobot. Two leg groups (A, B) must be used. The tripod of support is represented by the dash triangle.

\section{Control system}

The current 8-bits RISC architecture microcontroller design for this application does not support multiple voltages at the output parallel port [16]. While actuator can be modeled by a capacitor and a resistor [17], the use of PWM can adjust the level of $\mathrm{V}_{\text {RMS }}$. Then, the control of bending angle is possible. Finite State Machine (FSM) is designed with eight states and the output is controlled by PWM (Fig. 7). FSM has one input bit: F (Forward). The other MSB bits, R (Right) and L (Left), control the PWM width (duty cycle). The ten bits, including LSB $\alpha$ bits, is proportional to the bending angle of the actuator.

BMR will go forward when $\mathrm{R}$ and $\mathrm{L}$ are set to zero. Pulse width will be the same for the left and the right legs. Also, it can turn the same way as an insect when the duty cycle of the PWM is changed for the left or the right legs. Only three bits, stored in microcontroller LEG register, are used to control the direction of the robot. These bits can be modified by user algorithms.

\section{RESULtS}

Overall system is coded as a driver in VHDL and is tested on FPGA Xilinx Spartan IIE XC2S200 on a Digilab 2 (D2) board. Hardware test bench includes H-bridge MOSFET transistors to drive IPMC opposite electrodes on the ionomer and to isolate FPGA.

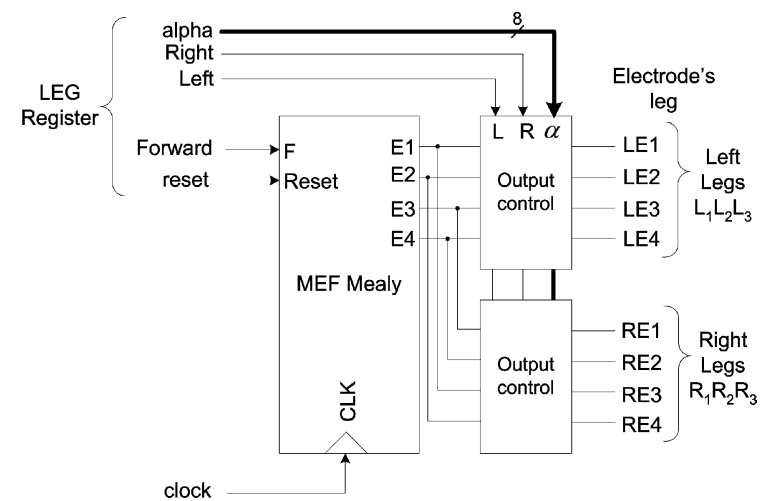

Fig. 7. FSM and output control driver inside the microcontroller. 


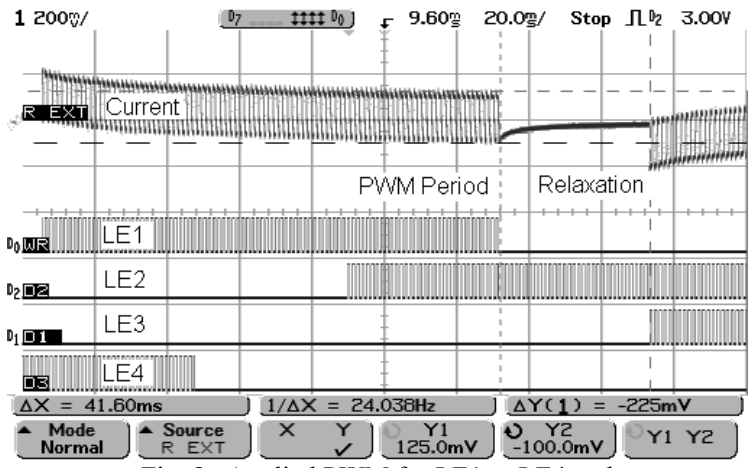

Fig. 8. Applied PWM for LE1 to LE4 and current consumption for LE1 and LE3

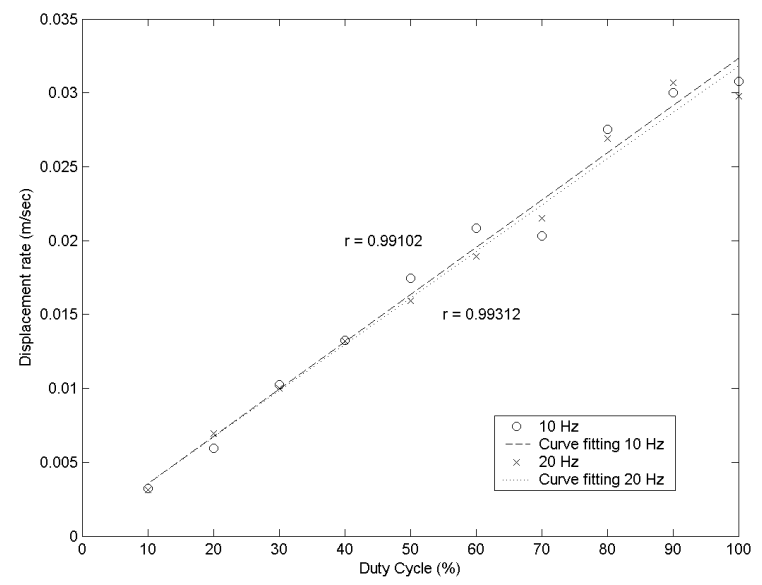

Fig. 9. Tip speed as a function of PWM duty cycle

Actually, PWM period is fixed in PWM module. Experimental results show that a frequency of $763 \mathrm{~Hz}$ and $24 \mathrm{~Hz}$ for PWM and FSM respectively can drive one BMR leg. Half period of stance is calculated at about 0,042 second (maximum bending to relaxation position). Fig. 8 shows FSM signal from FPGA and current consumption with timing parameters. Results shown in Fig. 9 are linear regressions of the maximum speed for the two frequencies. Actuator tip speed response deals with a great linearity in function of duty cycle. In future work, the module will be included in the microcontroller.

\section{CONCLUSION}

Using FSM and PWM to drive actuators, the robot can move in any direction similar to an insect with a specific configuration demonstrated in this paper. It has been shown that the tip speed of the actuator can be predicted by a linear equation of the duty cycle used to command the leg.

\section{ACKNOWLEDGMENT}

This work was partly support by Groupe d'acoustique et vibrations de l'Université de Sherbrooke (GAUS) and Institut des Matériaux et des Systèmes Intelligents (IMSI).
We thank Pierre Magny and Irène Levesque for advice and their suggestions to take SEM images of the composite. Also, we would like to thank Magella Tremblay and Patrice Masson for the use of its laboratory instrumentations.

\section{REFERENCES}

[1] K. E. Drexler, Engine of creation, the coming aera of nanotechnology, Doubleday, Broadway, New York, 1986, 298 p.

[2] A. Bonvilain, N. Chaillet, "Fabrication and Experiment of Microlegs for an Insect-Like Microrobot," Microrobotics and microassembly III, vol. 4568, p.163-174, 2001.

[3] K. Oguro, "3-Dimensional Dynamic Model of Ionic Conducting Polymer Gel Film (ICPF) actuator," Proc. of IEEE Int. Conf. on Systems, man and cybernetics, vol. 3, p. 2179-2184, Oct. 1996.

[4] S. Guo, T. Nakamura, T. Fukuda, "Micro active guide wire catheter system," Proc. of the IEEE International Conference on Industrial Technology (ICIT'96), Shanghai, China, p.517-521, Dec. 1996.

[5] K. Onishi, S. Sewa, K. Asaka, N. Fujiwara, K. Oguro, "The effect of counter ions on characterization and performance of a solid polymer electrolyte actuator," Electrochimica Acta, vol.46 no.8, p.1233-1241, Jan. 2001.

[6] K. Onishi, S. Sewa, K. Asaka, N. Fujiwara, K. Oguro, "Morphology of electrodes and bending response of the polymer electrolyte actuator," Electrochimica Acta, vol. 46, p.737-743 Dec. 2000.

[7] M. Shahinpoor, K.J. Kim, "The effect of surface-electrode resistance on the performance of ionic polymer-metal composite (IPMC) artificial muscles," Smart Materials and Structures, vol.9 no.4, p.543-551 Aug. 2000.

[8] Y. Bar-Cohen, Electroactive Polymer (EAP) Actuators as Artificial Muscles, Reality, Potential, and Challenges, US, Washington, SPIE Press, 671 p., 2001.

[9] G. Xie, T. Okada, "Pumping effects in water movement accompanying cation transport across nafion 117 membranes," Electrochimica Acta, vol. 41 - no. 9, p.1569-1571, May 1996.

[10] M. Shahinpoor, M. Mojarrad, "Soft actuators and artificial muscles", US patent 6109852, 18 p., Aug. 2000.

[11] S. Guo et al, "Micro Active Guide Wire Catheter System Characteristic Evaluation, Electrical Model and Operability Evaluation of MAC," Sixth International Symposium on Micro Machine and Human Science, Nagoya, Japan, p.131-136, Oct. 1995.

[12] H. Cruse, "What mechanisms coordinate leg movement in walking arthropods ?," Trends Neurosciences, vol. 13-1, pp. 1521, 1990.

[13] J. Gray, Animal locomotion, Great Britain, London and Beccles, The world naturalist/Editor: Richard Carrington, 479 p., 1968.

[14] L.H. Ting, R. Blickhan, R.-J. Full, "Dynamic and static stability in hexapedal runners," Journal of Experimental Biology, Vol. 197, No. 1, pp. 251-269, 1994.

[15] D.L. Jindrich, R.J. Full, "Many legged maneuvrability: dynamics of turning in hexapods," The Journal of Experimental Biology, Vol. 202, pp. 1603-1623, 1999.

[16] M. Jarry, M. Otis, H. Semmaoui, R. Fontaine, "Microcontroller architecture for autonomous BioMicroRobots Displacement Control," ReSMiQ's first Northeast Workshop on Circuits and Systems, Montreal, Canada, June 2003.

[17] X. Bao, Y. Bar-Cohen, S.S. Lih, "Measurements and macro models of ionomeric polymer-metal composites," Smart Structures and Materials 2002: Electroactive Polymer Actuators and Devices, San Diego, CA, USA, vol. 4695, p.220-227 March 2002. 